\title{
Socioeconomic Correlates of Obesity in African-American and Caribbean-Black Men and Women
}

\author{
Debbie S. Barrington ${ }^{1}$ (D) $\cdot$ Sherman A. James ${ }^{2} \cdot$ David R. Williams $^{3}$ \\ Received: 3 February 2020 / Revised: 29 May 2020 / Accepted: 5 June 2020 / Published online: 4 July 2020 \\ (C) W. Montague Cobb-NMA Health Institute 2020
}

\begin{abstract}
The high prevalence of obesity among Black Americans warrants additional investigation into its relationship with socioeconomic position (SEP), sex, and ethnicity. This cross-sectional study utilizes 2001-2003 data from the National Survey of American Life, a nationally representative sample of 3570 African-Americans and 1621 Caribbean-Blacks aged 18 years and older. Multivariate logistic regression models stratified by ethnicity and sex describe the independent associations between obesity and multilevel socioeconomic factors after adjustment for age, other SEP measures at the individual, family and neighborhood levels, and health behaviors such as physical activity, alcohol intake, and smoking. A positive relationship was observed between obesity and family income among African-American and Caribbean-Black men. Receipt of public assistance was a strongly associated factor for obesity in Caribbean-Black men and women. Among African-American women, inverse relationships were observed between obesity and education, occupation, and family income; residence within a neighborhood with a supermarket also decreased their odds of obesity. Residence in a neighborhood with a park decreased the odds of obesity only among African-American men, whereas residence in a neighborhood with a supermarket decreased the odds of obesity among Caribbean-Black men. The social patterning of obesity by individual, household, and neighborhood socioeconomic resources differs for African-American and Caribbean-Black men and women within these cross-sectional analyses; an appreciation of these differences may be a prerequisite for developing effective weight control interventions and policies for these two populations.
\end{abstract}

Keywords Adults $\cdot$ African American $\cdot$ Ethnic differences $\cdot$ Obesity $\cdot$ Socioeconomic

\section{Introduction}

The high prevalence of obesity among non-Hispanic Black Americans (38.3\%), aged 18 years and older in 2018, as

Debbie S. Barrington

debbie.barrington@georgetown.edu

Sherman A. James

sjames@duke.edu

David R. Williams

dwilliam@hsph.harvard.edu

1 Department of Human Science, Georgetown University, 237 St. Mary's Hall, 3700 Reservoir Road NW, Washington, DC 20057-1107, USA

2 Sanford School of Public Policy, Duke University, 203 Sanford Building, Durham, NC 27708-0245, USA

3 Department of Social and Behavioral Sciences, Harvard T.H. Chan School of Public Health, Harvard University, 677 Huntington Ave, Room 709, Boston, MA 02115, USA compared with $31.1 \%$ for the general US population, remains an important public health problem [1]. Obesity has a broad range of health consequences, and its higher prevalence among non-Hispanic Blacks puts them at increased risk for type 2 diabetes $($ Blacks $=13.0 \%$, Whites $=8.0 \%)$, coronary heart disease $($ Black women $=5.2 \%$, White women $=3.9 \%)$, asthma $($ Blacks $=9.2 \%$, Whites $=8.0 \%)$, and stroke $($ Blacks $=$ $4.0 \%$, Whites $=2.7 \%$ ) $[1]$.

The important role of socioeconomic position (SEP) in the obesity epidemic has long been a focus of research [2-5]. SEP is defined as one's location in the societal structure that determines differential access to power, privilege, and desirable resources [6]. It is a multidimensional concept that can be measured at (1) the individual level, e.g., education, earnings, and occupation; (2) the household level, characterized by familial resources, e.g., poverty, family income, and wealth; and (3) the neighborhood level, described by aspects of living conditions not captured by individual or household level variables, e.g., community structural characteristics, neighborhood poverty, and crime $[7,8]$. Prior research has documented 
correlations between lower rates of obesity and residence in communities with beneficial structural characteristics of neighborhood SEP, including the presence of neighborhood parks and supermarkets [9-11].

Research findings on the relationship between obesity and individual and household measures of SEP, however, have been more mixed. For example, some studies have revealed a weakening of the protective effect of increasing education on obesity over the past 30 years, a consequence of the increase in obesity among the most educated [12]. The inverse association between SEP and body mass index (BMI) and obesity is stronger among women compared with men $[2$, 13-17]. Some studies, in fact, reported a reversal of the expected inverse SEP-obesity association among men; i.e., men of high SEP were more likely to have increased BMI and be overweight or obese than men of low SEP [14-16, 18]. Furthermore, nativity status (foreign-born vs. US-born) differentials in the association of an individual-level measure of SEP on obesity have been reported among Black Americans, with native-born Blacks being at higher risk [19].

Few studies, however, have examined sex-specific associations between obesity and multilevel measures of SEP within an ethnically diverse group of Black Americans. Hence, the present study investigates the potential differential associations between obesity and individual, household, and neighborhood-level SEP indicators among Black American men and women (African-Americans) and Blacks with Caribbean ethnicity (Caribbean-Blacks). Elucidation of the differential patterning of multidimensional measures of SEP on obesity among African-American and Caribbean-Black men and women would be beneficial for developing effective interventions to reduce obesity and obesity-related diseases within an increasingly heterogeneous US Black population.

\section{Methods and Procedures}

This cross-sectional study utilizes data from the National Survey of American Life (NSAL). The NSAL is part of the National Institute of Mental Health (NIMH) Collaborative Psychiatric Epidemiology Surveys (CPES) initiative that also includes the National Co-morbidity Survey Replication (NCS-R) and the National Latino and Asian American Study (NLAAS) [20]. NSAL was conducted according to the guiding principles in the Declaration of Helsinki. All procedures involving human subjects, including oral and written consent from all participants 18 years of age and older, were approved by the Institutional Review Board at the University of Michigan [21]. The NSAL includes a sample of 891 nonHispanic Whites, 3570 African-Americans, persons who identified as Black but did not have ancestral ties to the Caribbean (including 67 foreign-born Blacks), and 1621 CaribbeanBlacks, persons who identified as Black and who either were born in Caribbean area countries ( $73 \%$ of the Caribbean-Black sample), had parents or grandparents who were born in the Caribbean, or had indicated that they were of Caribbean ethnicity. The NSAL survey data includes assessments of mental, emotional, and physical health and residential, environmental, and socioeconomic characteristics. Data were collected between February 2001 and June 2003 with response rates of $70.7 \%$ for African-Americans, $77.7 \%$ for Caribbean-Blacks, and $72.3 \%$ for Whites. This paper capitalizes on the growing heterogeneity of the US Black population as reflected in the NSAL sample and reports findings for African-American and Caribbean-Black men and women, aged 18 years and older within the NSAL sample.

The study outcome is obesity, characterized as having a body mass index (BMI, weight $(\mathrm{kg}) /$ height $\left.(\mathrm{m})^{2}\right) 30$ or higher [22]. BMI is calculated within the NSAL based on selfreported weight and height. The independent variables include self-reported measures of adult SEP at the individual, family, and neighborhood levels. SEP indicators at the individual level include current education specified as $<$ high school (HS), HS and $>$ HS, and respondent's main occupation categorized into $\mathrm{professional/managerial,} \mathrm{sales/administrative,} \mathrm{skilled}$ blue collar, unskilled blue collar, and service. SEP measures at the family level consist of (1) a measure of wealth or "house value," specified as at or below the median, above the median, or "none," (2) quartiles of family income, and (3) "public support" described as currently receiving any public assistance for the family, and dichotomized into "any" or "none." Finally, SEP measures assessed at the neighborhood level include self-reported measures previously found to be associated with obesity within African-Americans and CaribbeanBlacks - having a supermarket within one's neighborhood (yes vs. no) and having a park within one's neighborhood (yes vs. no) [23].

Covariates utilized for statistical adjustment due to their known associations with SEP and obesity include demographic variables such as age [5], categorized into less than or equal to 29 years, $30-44$ years, $45-59$ years, and 60 or more years; marital status, [24] characterized as (1) married or living with a partner, (2) separated, divorced or widowed, and (3) never married; and three measures of health behaviors, (1) physical activity [22], a continuous measure based on how often the study participants worked in the garden or yard, engaged in active sports or exercise, and walked, (2) smoking status [25], specified as the participant never having smoked more than 100 cigarettes in his/her lifetime or "never smoker," having smoked more than 100 cigarettes in the past or "past smoker," and "current smoker," and (3) current alcohol consumption [22], categorized for analytical purposes into consuming no alcoholic drinks within the past year or "none," having consumed less than 12 drinks within the past year, or "infrequent drinker," and moderate-to-heavy drinkers having consumed 12 or more drinks within the past year or "regular drinker." 
To minimize bias due to differentially distributed missing data on measures of SEP and BMI by ethnicity (AfricanAmerican and Caribbean-Black) and sex, multiple imputation was performed prior to statistical analysis within the statistical software package IVEware [26]. IVEware uses a sequential regression imputation method to impute values for each individual, conditional on all other values observed for that individual, consequently producing complete datasets for unbiased analyses [27]. Descriptive statistics stratified by ethnicity and by sex were then calculated. Chi-square tests were performed to examine group differences in categorical variables, whereas the global $F$ test within an unadjusted linear regression model was utilized to calculate ethnicity and sex differences in physical activity. Since previous studies reveal differential SEP patterning of obesity by both sex and ethnicity $[4,19]$, three-way interactions between measures of SEP, ethnicity, and sex were tested. Statistically significant three-way interactions were found for five out of the eight SEP indicators, $p$ values ranging from 0.008 to 0.024 . Therefore, all nested multivariate logistic regression models assessing the strength of the independent associations between multilevel measures of SEP and obesity after covariate adjustment are presented stratified by ethnicity and sex. Specifically, the following five models were fitted for each SEP indicator: (1) odds ratios (ORs) adjusted for age; (2) ORs additionally adjusted for individual-level SEP, i.e., education, occupation, as well as for marital status; (3) ORs additionally adjusted for family-level SEP, i.e., public support, house value, and family income; (4) ORs additionally adjusted for neighborhood-level SEP, i.e., neighborhood supermarket and neighborhood park; and (5) ORs additionally adjusted for health behaviors, i.e., physical activity, alcohol intake, and smoking.

Data management was conducted using SAS Version 9, [28] and all statistical analyses were performed using SUDAAN [29] to account for the multiple imputation and the complex sample design of the NSAL in calculating unbiased effect estimates and standard errors. Sample sizes presented within tables are un-weighted; however, all other estimates, means proportions, and standard errors, as well as ORs and $95 \%$ confidence intervals (CIs), are weighted.

\section{Results}

Table 1 describes the general characteristics of AfricanAmerican and Caribbean-Black men and women in the NSAL sample. African-American women had the highest prevalence of obesity at $41.2 \%$ followed by CaribbeanBlack women at $31.1 \%$ and African-American men at 29.9\%. Caribbean-Black men had the lowest prevalence of obesity at $21.6 \%$. Caribbean-Black men and women were also younger than African-American men and women with $64.9 \%$ and $66.2 \%$ of Caribbean-Black men and women being under the age of 45 as compared with $61.0 \%$ and $58.8 \%$ of AfricanAmerican men and women, respectively.

Caribbean-Black men and women had greater educational attainment than African-American men and women, with $49.4 \%$ of Caribbean-Black men and $48.8 \%$ of CaribbeanBlack women and $37.3 \%$ of African-American men and $38.4 \%$ of African-American women having > HS education. Men were more likely to be married or living with a partner than women; Caribbean-Black men had the highest prevalence at $59.9 \%$, while African-American women had the lowest at $35.6 \%$. In addition, men were more likely than women to be employed in the skilled and unskilled blue collar professions; unskilled blue collar employment was highest for African-American men at $33.2 \%$. Women were more likely than men to be employed in service professions. Moreover, Caribbean-Black men and women, at $23.0 \%$ and $23.3 \%$, respectively, were more likely to work in professional/ managerial positions compared with African-American men and women at $15.4 \%$ and $17.9 \%$, respectively.

African-American women were the most likely to receive public support, and accordingly, most likely to have had the lowest family income compared with all other ethnic/sex groups. Caribbean-Black men were least likely to receive public support and to have had the highest family income. African-American men were most likely to be homeowners relative to all other ethnic/sex groups, whereas CaribbeanBlack women were least likely to be homeowners.

Caribbean-Black men and women were more likely than African-American men and women to live in neighborhoods that had a supermarket or a park. Caribbean-Black men had the highest prevalence, in that $91 \%$ lived in a neighborhood with a supermarket and $88.1 \%$ resided in a neighborhood with a park. African-American women had the lowest prevalence with approximately $71 \%$ residing in a neighborhood that had a supermarket or park.

Men reported higher rates of physical activity than women, with Caribbean-Black men having the highest average score (2.95) and African-American women having the lowest (2.56). Seventy-nine percent of Caribbean-Black women never smoked, whereas only $49.4 \%$ of African-American men never smoked. Finally, African-American women were least likely to consume alcohol in the past year where $43.5 \%$ never drank. Caribbean-Black men were most likely to drink alcohol in the past year and $55.7 \%$ were regular drinkers.

Tables 2, 3, 4, and 5 present the adjusted odds ratios (ORs) and $95 \%$ confidence intervals for the associations between obesity and individual-, family-, and neighborhood-level socioeconomic position (SEP) among African-American men, Caribbean-Black men, African-American women, and Caribbean-Black women, respectively. Unless otherwise stated, all ORs were determined from the fully adjusted models.

For African-American men, no significant associations between obesity and any individual-level measure of SEP were 
Table 1 General characteristics of African-American and Caribbean-Black men and women: the National Survey of American Life

\begin{tabular}{|c|c|c|c|c|c|c|c|c|c|c|c|c|c|}
\hline & \multicolumn{3}{|c|}{ African-American men } & \multicolumn{3}{|c|}{ Caribbean-Black men } & \multicolumn{3}{|c|}{ African-American women } & \multicolumn{3}{|c|}{ Caribbean-Black women } & \multirow[t]{2}{*}{$P$ value } \\
\hline & $N$ & $\mathrm{Wt} \%$ & $\mathrm{Se}$ & $N$ & $\mathrm{Wt} \%$ & $\mathrm{Se}$ & $N$ & $\mathrm{Wt} \%$ & $\mathrm{Se}$ & $N$ & $\mathrm{Wt} \%$ & $\mathrm{Se}$ & \\
\hline Obesity & & & & & & & & & & & & & $<0.0001$ \\
\hline No & 903 & 70.10 & 1.34 & 528 & 78.41 & 4.45 & 1327 & 58.82 & 1.14 & 677 & 68.94 & 3.96 & \\
\hline Yes & 368 & 29.90 & 1.34 & 115 & 21.59 & 4.45 & 972 & 41.18 & 1.14 & 301 & 31.06 & 3.96 & \\
\hline Age & & & & & & & & & & & & & 0.0034 \\
\hline$\leq 29$ & 275 & 24.25 & 1.54 & 189 & 34.42 & 3.89 & 531 & 24.46 & 1.24 & 247 & 27.57 & 2.21 & \\
\hline $45-59$ & 324 & 23.85 & 1.34 & 134 & 20.00 & 3.81 & 531 & 23.74 & 0.97 & 222 & 18.69 & 2.36 & \\
\hline $60+$ & 224 & 15.10 & 1.14 & 102 & 15.08 & 3.58 & 409 & 17.42 & 1.12 & 122 & 15.10 & 2.19 & \\
\hline $30-44$ & 448 & 36.79 & 1.25 & 218 & 30.50 & 2.58 & 828 & 34.37 & 1.20 & 387 & 38.64 & 2.61 & \\
\hline Current education & & & & & & & & & & & & & 0.0013 \\
\hline$<\mathrm{HS}$ & 320 & 23.22 & 1.58 & 134 & 21.43 & 4.45 & 600 & 24.96 & 1.74 & 172 & 21.02 & 2.12 & \\
\hline HS & 497 & 39.45 & 1.78 & 194 & 29.16 & 3.59 & 865 & 36.61 & 1.30 & 287 & 30.15 & 2.80 & \\
\hline$>\mathrm{HS}$ & 454 & 37.33 & 2.10 & 315 & 49.42 & 3.86 & 834 & 38.44 & 1.57 & 519 & 48.83 & 3.54 & \\
\hline Marital status & & & & & & & & & & & & & $<0.0001$ \\
\hline Married/partner & 554 & 49.44 & 1.65 & 337 & 59.88 & 5.56 & 672 & 35.56 & 1.27 & 356 & 40.08 & 3.09 & \\
\hline Separated/divorced/widowed & 325 & 20.17 & 1.31 & 106 & 10.39 & 1.98 & 843 & 32.05 & 0.99 & 279 & 27.79 & 3.86 & \\
\hline Never married & 392 & 30.39 & 1.60 & 200 & 29.72 & 4.13 & 784 & 32.39 & 1.50 & 343 & 32.14 & 1.79 & \\
\hline Main occupation & & & & & & & & & & & & & $<0.0001$ \\
\hline Professional/Managerial & 179 & 15.44 & 1.44 & 130 & 22.98 & 4.09 & 403 & 17.88 & 1.14 & 240 & 23.36 & 3.10 & \\
\hline Sales/administrative & 193 & 15.61 & 1.47 & 136 & 23.03 & 5.17 & 694 & 32.25 & 1.19 & 284 & 28.92 & 2.97 & \\
\hline Blue Collar: skilled & 203 & 15.69 & 1.62 & 126 & 15.19 & 2.62 & 76 & 3.19 & 0.46 & 39 & 4.01 & 0.81 & \\
\hline Blue Collar: unskilled & 444 & 33.19 & 2.11 & 125 & 19.04 & 4.32 & 300 & 12.30 & 0.82 & 40 & 5.15 & 2.10 & \\
\hline Service & 252 & 20.07 & 1.37 & 126 & 19.77 & 3.82 & 827 & 34.38 & 1.73 & 375 & 38.56 & 3.38 & \\
\hline Public support & & & & & & & & & & & & & $<0.0001$ \\
\hline Any & 579 & 46.78 & 1.94 & 218 & 39.56 & 4.88 & 1488 & 63.45 & 1.61 & 472 & 55.75 & 3.09 & \\
\hline None & 692 & 53.22 & 1.94 & 425 & 60.44 & 4.88 & 811 & 36.55 & 1.61 & 506 & 44.25 & 3.09 & \\
\hline House value & & & & & & & & & & & & & 0.0005 \\
\hline At/below median & 254 & 19.45 & 1.59 & 59 & 12.98 & 2.75 & 457 & 19.10 & 1.48 & 75 & 12.52 & 2.84 & \\
\hline Above median & 404 & 34.28 & 2.13 & 216 & 33.13 & 3.97 & 575 & 27.07 & 1.66 & 288 & 29.43 & 3.31 & \\
\hline None & 613 & 46.27 & 2.46 & 368 & 53.89 & 3.90 & 1267 & 53.83 & 1.94 & 615 & 58.05 & 2.74 & \\
\hline Family income & & & & & & & & & & & & & $<0.0001$ \\
\hline $0-25 \%$ & 277 & 18.45 & 1.52 & 82 & 13.19 & 3.74 & 777 & 29.61 & 1.42 & 191 & 17.95 & 2.48 & \\
\hline $26-50 \%$ & 309 & 22.21 & 1.30 & 145 & 21.24 & 2.87 & 690 & 28.33 & 1.24 & 275 & 28.20 & 3.08 & \\
\hline $51-75 \%$ & 374 & 29.97 & 1.38 & 184 & 24.02 & 3.53 & 474 & 21.76 & 1.00 & 258 & 24.82 & 2.08 & \\
\hline $76-100 \%$ & 311 & 29.37 & 2.11 & 232 & 41.55 & 4.73 & 358 & 20.29 & 1.54 & 254 & 29.02 & 3.50 & \\
\hline Supermarket in neighborhood & & & & & & & & & & & & & $<0.0001$ \\
\hline Yes & 940 & 75.66 & 1.91 & 586 & 91.04 & 2.37 & 1606 & 70.95 & 1.74 & 869 & 86.82 & 2.26 & \\
\hline No & 331 & 24.34 & 1.91 & 57 & 8.96 & 2.37 & 693 & 29.05 & 1.74 & 109 & 13.18 & 2.26 & \\
\hline Park in neighborhood & & & & & & & & & & & & & $<0.0001$ \\
\hline Yes & 945 & 76.63 & 1.94 & 544 & 88.06 & 2.57 & 1560 & 70.57 & 1.59 & 827 & 85.23 & 2.26 & \\
\hline No & 326 & 23.37 & 1.94 & 99 & 11.94 & 2.57 & 739 & 29.43 & 1.59 & 151 & 14.77 & 2.26 & \\
\hline Mean physical activity & 1271 & 2.88 & 0.03 & 643 & 2.95 & 0.07 & 2299 & 2.56 & 0.02 & 978 & 2.64 & 0.05 & 0.0053 \\
\hline Smoking status & & & & & & & & & & & & & $<0.0001$ \\
\hline Never & 610 & 49.42 & 1.65 & 427 & 63.80 & 3.71 & 1439 & 62.46 & 1.44 & 783 & 79.02 & 3.19 & \\
\hline Past & 253 & 19.09 & 1.20 & 89 & 16.37 & 3.93 & 312 & 13.72 & 1.10 & 93 & 8.59 & 1.87 & \\
\hline Current & 408 & 31.49 & 1.77 & 127 & 19.83 & 3.80 & 548 & 23.82 & 1.22 & 102 & 12.39 & 3.41 & \\
\hline Current alcohol consumption & & & & & & & & & & & & & $<0.0001$ \\
\hline None & 374 & 28.70 & 2.09 & 182 & 21.85 & 4.07 & 1006 & 43.51 & 1.96 & 436 & 39.38 & 3.89 & \\
\hline Infrequent & 236 & 19.27 & 1.13 & 132 & 22.42 & 2.12 & 559 & 23.64 & 1.56 & 269 & 29.87 & 2.79 & \\
\hline Regular & 661 & 52.03 & 2.06 & 329 & 55.73 & 4.94 & 734 & 32.85 & 1.33 & 273 & 30.75 & 3.12 & \\
\hline
\end{tabular}

${ }^{\text {a }} P$ values are based on Chi-square and F-tests examining group differences by ethnicity and sex

observed (Table 2). Low SEP at the family level, however, was associated with a lower odds of obesity. Within all adjusted models for African-American men, family income within the lowest quartile was protective against obesity relative to the highest quartile, $\mathrm{OR}=0.50,95 \% \mathrm{CI}(0.27,0.92)$. Furthermore, African-American male homeowners with housing equity at or below the median had a $43 \%$ increased odds of obesity compared with their non-home-owning counterparts, $\mathrm{OR}=1.43,95 \%$ CI $(1.00,2.06)$. Neighborhood-level SEP was also associated with obesity, with all adjusted models showing lower odds of obesity for African-American men residing in neighborhoods that contained a park compared with those who lived in neighborhoods without one $(\mathrm{OR}=$ $0.60,95 \%$ CI $(0.38,0.94))$. 
Table 2 Unadjusted and adjusted odds ratios for obesity among African-American men: the National Survey of American Life

\begin{tabular}{|c|c|c|c|c|c|c|c|c|c|c|}
\hline \multirow[t]{2}{*}{ SEP measure } & \multicolumn{2}{|c|}{ Age adjusted } & \multicolumn{2}{|c|}{$\begin{array}{l}+ \text { individual-level SEP } \\
\text { and marital status }\end{array}$} & \multicolumn{2}{|c|}{+ family-level SEP ${ }^{\mathrm{b}}$} & \multicolumn{2}{|c|}{$\begin{array}{l}+ \text { neighborhood-level } \\
\text { SEP }^{\mathrm{c}}\end{array}$} & \multicolumn{2}{|c|}{+ health behaviors ${ }^{\mathrm{d}}$} \\
\hline & Odds ratio & $95 \% \mathrm{CI}$ & Odds ratio & $95 \% \mathrm{CI}$ & Odds ratio & $95 \% \mathrm{CI}$ & Odds ratio & $95 \% \mathrm{CI}$ & Odds ratio & $95 \% \mathrm{CI}$ \\
\hline \multicolumn{11}{|l|}{ Current education } \\
\hline$<\mathrm{HS}$ & 0.92 & $(0.65,1.32)$ & 0.99 & $(0.65,1.51)$ & 1.17 & $(0.73,1.87)$ & 1.13 & $(0.69,1.83)$ & 1.18 & $(0.71,1.96)$ \\
\hline HS & 0.87 & $(0.65,1.17)$ & 0.90 & $(0.64,1.28)$ & 0.96 & $(0.65,1.43)$ & 0.94 & $(0.63,1.39)$ & 0.93 & $(0.62,1.41)$ \\
\hline$>\mathrm{HS}$ & REF & REF & REF & REF & REF & REF & REF & REF & REF & REF \\
\hline \multicolumn{11}{|l|}{ Main occupation } \\
\hline Professional/managerial & 1.63 & $(0.93,2.87)$ & 1.53 & $(0.82,2.85)$ & 1.45 & $(0.76,2.79)$ & 1.42 & $(0.73,2.74)$ & 1.37 & $(0.71,2.61)$ \\
\hline Sales/Administrative & 1.61 & $(0.95,2.71)$ & 1.62 & $(0.96,2.73)$ & 1.55 & $(0.90,2.67)$ & 1.53 & $(0.90,2.62)$ & 1.55 & $(0.92,2.63)$ \\
\hline Blue collar: skilled & 1.43 & $(0.75,2.74)$ & 1.43 & $(0.74,2.77)$ & 1.37 & $(0.68,2.76)$ & 1.36 & $(0.68,2.72)$ & 1.49 & $(0.77,2.91)$ \\
\hline Blue collar: unskilled & 1.53 & $(0.95,2.47)$ & 1.52 & $(0.93,2.48)$ & 1.51 & $(0.90,2.55)$ & 1.47 & $(0.87,2.46)$ & 1.53 & $(0.91,2.56)$ \\
\hline Service & REF & REF & REF & REF & REF & REF & REF & REF & REF & REF \\
\hline \multicolumn{11}{|l|}{ Public support } \\
\hline Any & 0.96 & $(0.75,1.22)$ & 0.99 & $(0.77,1.27)$ & 1.04 & $(0.80,1.37)$ & 1.06 & $(0.81,1.39)$ & 1.03 & $(0.78,1.37)$ \\
\hline None & REF & REF & REF & REF & REF & REF & REF & REF & REF & REF \\
\hline \multicolumn{11}{|l|}{ House value } \\
\hline At/below median & 1.70 & $(1.17,2.46)$ & 1.58 & $(1.10,2.29)$ & 1.46 & $(1.00,2.12)$ & 1.37 & $(0.95,1.98)$ & 1.43 & $(1.00,2.06)$ \\
\hline Above median & 1.40 & $(0.94,2.09)$ & 1.29 & $(0.85,1.96)$ & 1.17 & $(0.76,1.79)$ & 1.13 & $(0.75,1.72)$ & 1.13 & $(0.74,1.73)$ \\
\hline None & REF & REF & REF & REF & REF & REF & REF & REF & REF & REF \\
\hline \multicolumn{11}{|l|}{ Family income } \\
\hline $0-25 \%$ & 0.45 & $(0.29,0.71)$ & 0.47 & $(0.27,0.80)$ & 0.51 & $(0.28,0.91)$ & 0.47 & $(0.26,0.85)$ & 0.50 & $(0.27,0.92)$ \\
\hline $26-50 \%$ & 0.73 & $(0.45,1.18)$ & 0.77 & $(0.44,1.35)$ & 0.82 & $(0.46,1.45)$ & 0.75 & $(0.41,1.37)$ & 0.79 & $(0.43,1.46)$ \\
\hline $51-75 \%$ & 0.78 & $(0.55,1.11)$ & 0.81 & $(0.55,1.19)$ & 0.86 & $(0.58,1.27)$ & 0.83 & $(0.56,1.25)$ & 0.85 & $(0.56,1.30)$ \\
\hline $76-100 \%$ & REF & REF & REF & REF & REF & REF & REF & REF & REF & REF \\
\hline \multicolumn{11}{|c|}{ Supermarket in neighborhood } \\
\hline Yes & 0.92 & $(0.69,1.22)$ & 0.89 & $(0.67,1.18)$ & 0.90 & $(0.67,1.23)$ & 1.02 & $(0.73,1.43)$ & 1.03 & $(0.73,1.45)$ \\
\hline No & REF & REF & REF & REF & REF & REF & REF & REF & REF & REF \\
\hline \multicolumn{11}{|l|}{ Park in neighborhood } \\
\hline Yes & 0.62 & $(0.42,0.92)$ & 0.61 & $(0.40,0.92)$ & 0.59 & $(0.39,0.91)$ & 0.59 & $(0.38,0.92)$ & 0.60 & $(0.38,0.94)$ \\
\hline No & REF & REF & REF & REF & REF & REF & REF & REF & REF & REF \\
\hline
\end{tabular}

${ }^{a}$ Each SEP measure additionally adjusted for marital status, education, and/or occupation

${ }^{\mathrm{b}}$ Each SEP measure additionally adjusted for public support, house value, and/or family income

${ }^{\mathrm{c}}$ Each SEP measure additionally adjusted for neighborhood supermarket and/or park

${ }^{\mathrm{d}}$ Each SEP measure additionally adjusted for physical activity, smoking, and alcohol intake

As shown in Table 3, none of the individual-level measures of SEP for Caribbean-Black men was significantly associated with obesity, paralleling the findings for African-American men. Nevertheless, selected family- and neighborhood-level SEP measures were associated with obesity. For example, like African-American men, low family income was protective for Caribbean-Black men within the age-adjusted model; however, this association became non-significant $(P>0.05)$ with additional covariate adjustment. Furthermore, Caribbean-Black men who received any public assistance had 3.5 times the odds $(95 \%$ CI $1.30,9.42)$ of obesity compared with their counterparts who did not. Finally, Caribbean-Black men residing in a neighborhood containing a supermarket experienced an $86 \%$ decreased odds of obesity compared with those in neighborhoods without this amenity, $\mathrm{OR}=0.14,95 \% \mathrm{CI}$ $(0.04,0.52)$.

In contrast to men, Table 4 shows statistically significant associations between measures of individual-level SEP and obesity for African-American women. African-American women with $<$ HS education had 1.47 times the odds $(95 \%$ CI $(1.04,2.09))$ of obesity than those with $>$ HS education. Though only borderline significant in fully adjusted models, African-American women with a HS education had 1.40 times the odds $(95 \% \mathrm{CI}(0.96,2.05))$ of obesity compared with those with $>$ HS education. African-American women employed in the sales/administrative occupations had 27\% lower odds of obesity compared with African-American women employed in service employment, $\mathrm{OR}=0.73,95 \% \mathrm{CI}(0.57,0.94)$.

In contrast to African-American men, higher family SEP was protective against obesity for African-American women. Though of borderline significance, African-American women who received public support had a $27 \%$ greater odds of obesity compared with those not receiving such support, $\mathrm{OR}=$ $1.27,95 \% \mathrm{CI}(0.98,1.63)$. Though not statistically significant, African-American women with family income in the third quartile were associated with a $35 \%$ increased odds of obesity compared with women in the highest quartile, $\mathrm{OR}=1.35$, $95 \%$ CI $(0.97,1.89)$. African-American females with housing 
Table 3 Unadjusted and adjusted odds ratios for obesity among Caribbean-Black men: the National Survey of American Life

\begin{tabular}{|c|c|c|c|c|c|c|c|c|c|c|}
\hline \multirow[t]{2}{*}{ SEP measure } & \multicolumn{2}{|c|}{ Age adjusted } & \multicolumn{2}{|c|}{$\begin{array}{l}\text { + individual-level SEP } \\
\text { and marital status }^{\mathrm{a}}\end{array}$} & \multicolumn{2}{|c|}{+ family-level SEP ${ }^{\mathrm{b}}$} & \multicolumn{2}{|c|}{$\begin{array}{l}\text { + neighborhood-level } \\
\text { SEP }^{\mathrm{c}}\end{array}$} & \multicolumn{2}{|c|}{+ health behaviors ${ }^{\mathrm{d}}$} \\
\hline & Odds ratio & $95 \% \mathrm{CI}$ & Odds ratio & $95 \% \mathrm{CI}$ & Odds ratio & $95 \% \mathrm{CI}$ & Odds ratio & $95 \% \mathrm{CI}$ & Odds ratio & $95 \% \mathrm{CI}$ \\
\hline \multicolumn{11}{|l|}{ Current education } \\
\hline$<\mathrm{HS}$ & 0.28 & $(0.07,1.16)$ & 0.30 & $(0.08,1.15)$ & 0.45 & $(0.13,1.53)$ & 0.49 & $(0.13,1.83)$ & 0.58 & $(0.16,2.12$ \\
\hline HS & 0.69 & $(0.20,2.32)$ & 0.80 & $(0.19,3.28)$ & 0.78 & $(0.23,2.63)$ & 0.84 & $(0.20,3.47)$ & 0.84 & $(0.28,2.50$ \\
\hline$>\mathrm{HS}$ & REF & REF & REF & REF & REF & REF & REF & REF & REF & REF \\
\hline \multicolumn{11}{|l|}{ Main occupation } \\
\hline Professional/managerial & 2.24 & $(0.56,8.87)$ & 1.82 & $(0.38,8.64)$ & 1.88 & $(0.60,5.85)$ & 1.66 & $(0.54,5.18)$ & 1.70 & $(0.67,4.31)$ \\
\hline Sales/administrative & 1.08 & $(0.36,3.19)$ & 0.89 & $(0.32,2.49)$ & 0.95 & $(0.42,2.14)$ & 1.04 & $(0.47,2.27)$ & 0.84 & $(0.34,2.07$ \\
\hline Blue collar: skilled & 0.55 & $(0.15,2.09)$ & 0.54 & $(0.16,1.78)$ & 0.46 & $(0.15,1.47)$ & 0.47 & $(0.14,1.56)$ & 0.54 & $(0.20,1.45$ \\
\hline Blue collar: unskilled & 1.45 & $(0.37,5.72)$ & 1.99 & $(0.66,6.04)$ & 1.92 & $(0.66,5.56)$ & 1.48 & $(0.58,3.79)$ & 1.45 & $(0.57,3.72$ \\
\hline Service & REF & REF & REF & $\mathrm{REF}$ & REF & REF & REF & REF & REF & REF \\
\hline \multicolumn{11}{|l|}{ Public support } \\
\hline Any & 2.15 & $(0.77,5.99)$ & 2.78 & $(1.00,7.76)$ & 2.65 & $(1.00,7.03)$ & 3.18 & $(1.19,8.50)$ & 3.50 & $(1.30,9.42$ \\
\hline None & REF & REF & REF & REF & REF & REF & REF & REF & REF & REF \\
\hline \multicolumn{11}{|l|}{ House value } \\
\hline At/below median & 2.17 & $(0.33,14.35)$ & 1.41 & $(0.37,5.34)$ & 1.23 & $(0.34,4.45)$ & 1.26 & $(0.39,4.05)$ & 0.90 & $(0.34,2.38$ \\
\hline Above median & 1.12 & $(0.60,2.08)$ & 0.97 & $(0.57,1.65)$ & 0.85 & $(0.47,1.53)$ & 0.86 & $(0.46,1.62)$ & 0.60 & $(0.26,1.39$ \\
\hline None & REF & REF & REF & REF & REF & REF & REF & REF & REF & REF \\
\hline \multicolumn{11}{|l|}{ Family income } \\
\hline $0-25 \%$ & 0.19 & $(0.04,0.94)$ & 0.27 & $(0.05,1.36)$ & 0.24 & $(0.04,1.30)$ & 0.29 & $(0.05,1.72)$ & 0.24 & $(0.04,1.39$ \\
\hline $26-50 \%$ & 0.44 & $(0.12,1.55)$ & 0.56 & $(0.20,1.57)$ & 0.53 & $(0.17,1.62)$ & 0.55 & $(0.17,1.82)$ & 0.47 & $(0.13,1.76$ \\
\hline $51-75 \%$ & 0.92 & $(0.40,2.10)$ & 1.09 & $(0.54,2.23)$ & 1.00 & $(0.46,2.17)$ & 0.96 & $(0.44,2.11)$ & 0.87 & $(0.37,2.06$ \\
\hline $76-100 \%$ & REF & REF & REF & REF & REF & REF & REF & REF & REF & REF \\
\hline \multicolumn{11}{|c|}{ Supermarket in neighborhood } \\
\hline Yes & 0.19 & $(0.07,0.51)$ & 0.16 & $(0.05,0.52)$ & 0.14 & $(0.04,0.50)$ & 0.14 & $(0.04,0.48)$ & 0.14 & $(0.04,0.52$ \\
\hline No & REF & REF & REF & REF & REF & REF & REF & REF & REF & REF \\
\hline \multicolumn{11}{|l|}{ Park in neighborhood } \\
\hline Yes & 0.83 & $(0.35,1.95)$ & 0.69 & $(0.22,2.17)$ & 0.63 & $(0.17,2.23)$ & 1.00 & $(0.44,2.25)$ & 0.91 & $(0.34,2.40$ \\
\hline No & REF & REF & REF & REF & REF & REF & REF & REF & REF & REF \\
\hline
\end{tabular}

${ }^{a}$ Each SEP measure additionally adjusted for marital status, education, and/or occupation

${ }^{\mathrm{b}}$ Each SEP measure additionally adjusted for public support, house value, and/or family income

${ }^{\mathrm{c}}$ Each SEP measure additionally adjusted for neighborhood supermarket and/or park

${ }^{\mathrm{d}}$ Each SEP measure additionally adjusted for physical activity, smoking, and alcohol intake

equity above the median had $22 \%$ lower odds of obesity compared with their non-home-owning counterparts, though borderline significant, $\mathrm{OR}=0.78,95 \% \mathrm{CI}(0.59,1.03)$. Finally, at the neighborhood level, African-American women residing in neighborhoods containing a supermarket had a $26 \%$ decreased odds of obesity compared with those without this amenity, $\mathrm{OR}=0.74,95 \% \mathrm{CI}(0.56,0.96)$.

Table 5 summarizes associations between SEP and obesity among Caribbean-Black women. Occupation is the only individual-level SEP measure that was associated with obesity in this group. Being employed within professional/managerial occupations was associated with a decreased odds of obesity compared with employment in service occupations, after adjusting for age, marital status, and other individual-level, family-level, and neighborhood-level SEP measures, $\mathrm{OR}=0.55$, $95 \%$ CI $(0.30,0.99)$. This association became statistically insignificant, however, after adjusting for physical activity, smoking, and alcohol consumption, $\mathrm{OR}=0.61,95 \% \mathrm{CI}(0.35$, 1.07).

Similar to African-American women, receipt of public assistance increased the odds of obesity for Caribbean-Black women; however, the magnitude of the association was stronger for the latter. Caribbean-Black women receiving public support had 1.74 times the odds of obesity compared with Caribbean-Black women not on public assistance, 95\% CI $(1.08,2.82)$. Yet, unlike African-American women, in ageadjusted and individual-level SEP adjusted models, Caribbean-Black women with family income in the third quartile had a decreased odds of obesity compared with those with family income in the fourth quartile. In fully adjusted models, this relationship became statistically insignificant, $\mathrm{OR}=0.56$, $95 \%$ CI $(0.28,1.14)$. No association was observed between 
Table 4 Unadjusted and adjusted odds ratios for obesity among African-American women: the National Survey of American Life

\begin{tabular}{|c|c|c|c|c|c|c|c|c|c|c|}
\hline \multirow[t]{2}{*}{ SEP Measure } & \multicolumn{2}{|c|}{ Age adjusted } & \multicolumn{2}{|c|}{$\begin{array}{l}+ \text { individual-level SEP } \\
\text { and marital status }{ }^{\mathrm{a}}\end{array}$} & \multicolumn{2}{|c|}{ + family-level SEP ${ }^{\mathrm{b}}$} & \multicolumn{2}{|c|}{$\begin{array}{l}+ \text { neighborhood-level } \\
\text { SEP }^{\mathrm{c}}\end{array}$} & \multicolumn{2}{|c|}{+ health behaviors ${ }^{\mathrm{d}}$} \\
\hline & Odds ratio & $95 \% \mathrm{CI}$ & Odds ratio & $95 \% \mathrm{CI}$ & Odds ratio & $95 \% \mathrm{CI}$ & Odds ratio & $95 \% \mathrm{CI}$ & Odds ratio & $95 \% \mathrm{CI}$ \\
\hline \multicolumn{11}{|l|}{ Current education } \\
\hline$<\mathrm{HS}$ & 1.81 & $(1.39,2.36)$ & 1.62 & $(1.19,2.20)$ & 1.49 & $(1.05,2.09)$ & 1.42 & $(1.01,2.00)$ & 1.47 & $(1.04,2.09)$ \\
\hline HS & 1.59 & $(1.17,2.15)$ & 1.51 & $(1.08,2.13)$ & 1.44 & $(1.00,2.07)$ & 1.42 & $(0.98,2.05)$ & 1.40 & $(0.96,2.05)$ \\
\hline$>\mathrm{HS}$ & REF & REF & REF & REF & REF & REF & REF & REF & REF & REF \\
\hline \multicolumn{11}{|l|}{ Main occupation } \\
\hline Professional/managerial & 0.61 & $(0.44,0.86)$ & 0.76 & $(0.53,1.09)$ & 0.81 & $(0.57,1.17)$ & 0.82 & $(0.57,1.19)$ & 0.80 & $(0.55,1.18)$ \\
\hline Sales/administrative & 0.65 & $(0.51,0.83)$ & 0.72 & $(0.57,0.92)$ & 0.73 & $(0.58,0.93)$ & 0.74 & $(0.59,0.94)$ & 0.73 & $(0.57,0.94)$ \\
\hline Blue collar: skilled & 0.66 & $(0.36,1.23)$ & 0.76 & $(0.41,1.40)$ & 0.82 & $(0.43,1.55)$ & 0.83 & $(0.44,1.56)$ & 0.81 & $(0.42,1.58)$ \\
\hline Blue collar: unskilled & 0.92 & $(0.68,1.25)$ & 0.91 & $(0.66,1.23)$ & 0.92 & $(0.67,1.26)$ & 0.90 & $(0.66,1.24)$ & 0.98 & $(0.71,1.35)$ \\
\hline Service & REF & REF & REF & REF & REF & REF & REF & REF & REF & REF \\
\hline \multicolumn{11}{|l|}{ Public support } \\
\hline Any & 1.38 & $(1.07,1.78)$ & 1.28 & $(0.99,1.67)$ & 1.27 & $(0.99,1.63)$ & 1.25 & $(0.98,1.61)$ & 1.27 & $(0.98,1.63)$ \\
\hline None & REF & REF & REF & REF & REF & REF & REF & REF & REF & REF \\
\hline \multicolumn{11}{|l|}{ House value } \\
\hline At/below median & 1.01 & $(0.81,1.27)$ & 1.04 & $(0.83,1.28)$ & 1.08 & $(0.86,1.35)$ & 1.00 & $(0.81,1.24)$ & 1.01 & $(0.79,1.28)$ \\
\hline Above median & 0.73 & $(0.57,0.94)$ & 0.79 & $(0.61,1.02)$ & 0.82 & $(0.63,1.08)$ & 0.78 & $(0.60,1.03)$ & 0.78 & $(0.59,1.03)$ \\
\hline None & REF & REF & REF & REF & REF & REF & REF & REF & REF & REF \\
\hline \multicolumn{11}{|l|}{ Family income } \\
\hline $0-25 \%$ & 1.58 & $(1.15,2.16)$ & 1.30 & $(0.87,1.96)$ & 1.15 & $(0.76,1.75)$ & 1.12 & $(0.74,1.69)$ & 1.19 & $(0.77,1.84)$ \\
\hline $26-50 \%$ & 1.51 & $(1.09,2.09)$ & 1.33 & $(0.90,1.98)$ & 1.26 & $(0.83,1.89)$ & 1.22 & $(0.81,1.83)$ & 1.24 & $(0.80,1.90)$ \\
\hline $51-75 \%$ & 1.47 & $(1.07,2.01)$ & 1.38 & $(0.98,1.94)$ & 1.31 & $(0.93,1.85)$ & 1.30 & $(0.93,1.81)$ & 1.35 & $(0.97,1.89)$ \\
\hline $76-100 \%$ & REF & REF & REF & REF & REF & REF & REF & REF & REF & REF \\
\hline \multicolumn{11}{|c|}{ Supermarket in neighborhood } \\
\hline Yes & 0.68 & $(0.54,0.86)$ & 0.73 & $(0.57,0.93)$ & 0.72 & $(0.57,0.92)$ & 0.73 & $(0.56,0.94)$ & 0.74 & $(0.56,0.96)$ \\
\hline No & REF & REF & REF & REF & REF & REF & REF & REF & REF & REF \\
\hline \multicolumn{11}{|l|}{ Park in neighborhood } \\
\hline Yes & 0.80 & $(0.66,0.98)$ & 0.88 & $(0.72,1.07)$ & 0.90 & $(0.75,1.08)$ & 0.94 & $(0.77,1.14)$ & 1.00 & $(0.82,1.23)$ \\
\hline No & REF & REF & REF & REF & REF & REF & REF & REF & REF & REF \\
\hline
\end{tabular}

${ }^{a}$ Each SEP measure additionally adjusted for marital status, education, and/or occupation

${ }^{\mathrm{b}}$ Each SEP measure additionally adjusted for public support, house value, and/or family income

${ }^{\mathrm{c}}$ Each SEP measure additionally adjusted for neighborhood supermarket and/or park

${ }^{\mathrm{d}}$ Each SEP measure additionally adjusted for physical activity, smoking, and alcohol intake

obesity and neighborhood-level SEP variables for CaribbeanBlack women, in contrast to findings for both groups of men.

\section{Discussion}

This study of the association between multiple dimensions of SEP (individual, family, and neighborhood) and obesity in a sample of US Blacks found that associations varied by ethnicity and by sex. Among African-American men, no statistically significant associations between SEP and obesity were observed at the individual level, but a positive relationship was observed for family SEP; namely, African-American men in the bottom quartile of family income and who had no housing equity had the lowest odds of obesity. Similar to AfricanAmerican men, there was no association between adulthood SEP at the individual level among Caribbean-Black men. For Caribbean-Black men, more complex SEP patterns for family income were observed, wherein (1) obesity increased among those receiving public assistance and (2), similar to AfricanAmerican men, a positive association was observed between family income and obesity.

The association between public assistance and the increasing odds of obesity among Caribbean-Black men is consistent with a previous report showing that participation in public assistance programs increased the risk of adult obesity [30]. In this particular study, the association was stronger among men than women and was mediated by dietary quality as assessed by higher soda consumption. Moreover, in the current study, our finding of a positive association between obesity and SEP at the family level among Black men is also in line with previous reports $[5,14,31,32]$. African-American elementary school boys with low SEP have been reported to engage in more vigorous physical activity than high SEP boys through participation in team sports such as football and basketball [33]. This engagement in vigorous team sports, if it 
Table 5 Unadjusted and adjusted odds ratios for obesity among Caribbean-Black women: the National Survey of American Life

\begin{tabular}{|c|c|c|c|c|c|c|c|c|c|c|}
\hline \multirow[t]{2}{*}{ SEP measure } & \multicolumn{2}{|c|}{ Age adjusted } & \multicolumn{2}{|c|}{$\begin{array}{l}+ \text { individual-level SEP } \\
\text { and marital status }\end{array}$} & \multicolumn{2}{|c|}{+ family-level SEP ${ }^{\mathrm{b}}$} & \multicolumn{2}{|c|}{$\begin{array}{l}+ \text { neighborhood-level } \\
\text { SEP }^{\mathrm{c}}\end{array}$} & \multicolumn{2}{|c|}{+ health behaviors ${ }^{\mathrm{d}}$} \\
\hline & Odds ratio & $95 \% \mathrm{CI}$ & Odds ratio & $95 \% \mathrm{CI}$ & Odds ratio & $95 \% \mathrm{CI}$ & Odds ratio & $95 \% \mathrm{CI}$ & Odds ratio & $95 \% \mathrm{CI}$ \\
\hline \multicolumn{11}{|l|}{ Current education } \\
\hline$<\mathrm{HS}$ & 1.02 & $(0.42,2.45)$ & 0.84 & $(0.37,1.88)$ & 0.84 & $(0.44,1.61)$ & 0.80 & $(0.42,1.53)$ & 0.78 & $(0.41,1.49)$ \\
\hline HS & 1.00 & $(0.48,2.07)$ & 0.83 & $(0.40,1.75)$ & 0.86 & $(0.49,1.51)$ & 0.84 & $(0.49,1.42)$ & 0.84 & $(0.47,1.52)$ \\
\hline$>\mathrm{HS}$ & REF & REF & REF & REF & REF & REF & REF & REF & REF & REF \\
\hline \multicolumn{11}{|l|}{ Main occupation } \\
\hline Professional/managerial & 0.65 & $(0.32,1.31)$ & 0.55 & $(0.29,1.05)$ & 0.54 & $(0.30,0.97)$ & 0.55 & $(0.30,0.99)$ & 0.61 & $(0.35,1.07)$ \\
\hline Sales/administrative & 0.79 & $(0.37,1.69)$ & 0.72 & $(0.33,1.53)$ & 0.70 & $(0.35,1.39)$ & 0.70 & $(0.35,1.40)$ & 0.66 & $(0.33,1.32)$ \\
\hline Blue collar: skilled & 0.49 & $(0.13,1.93)$ & 0.46 & $(0.12,1.78)$ & 0.42 & $(0.10,1.75)$ & 0.42 & $(0.10,1.76)$ & 0.36 & $(0.10,1.25)$ \\
\hline Blue collar: unskilled & 0.35 & $(0.11,1.16)$ & 0.38 & $(0.12,1.26)$ & 0.35 & $(0.10,1.20)$ & 0.34 & $(0.10,1.17)$ & 0.41 & $(0.11,1.50)$ \\
\hline Service & REF & REF & REF & REF & REF & REF & REF & REF & REF & REF \\
\hline \multicolumn{11}{|l|}{ Public support } \\
\hline Any & 1.91 & $(1.18,3.09)$ & 2.09 & $(1.32,3.32)$ & 1.91 & $(1.27,2.87)$ & 1.91 & $(1.25,2.89)$ & 1.74 & $(1.08,2.82)$ \\
\hline None & REF & REF & REF & REF & REF & REF & REF & REF & REF & REF \\
\hline \multicolumn{11}{|l|}{ House value } \\
\hline At/below median & 1.31 & $(0.33,5.28)$ & 1.31 & $(0.40,4.34)$ & 1.22 & $(0.38,3.87)$ & 1.26 & $(0.39,4.08)$ & 1.51 & $(0.51,4.50)$ \\
\hline Above median & 0.93 & $(0.52,1.65)$ & 0.92 & $(0.55,1.55)$ & 0.91 & $(0.51,1.61)$ & 0.90 & $(0.51,1.62)$ & 1.17 & $(0.62,2.22)$ \\
\hline None & REF & REF & REF & REF & REF & REF & REF & REF & REF & REF \\
\hline \multicolumn{11}{|l|}{ Family income } \\
\hline $0-25 \%$ & 0.99 & $(0.30,3.24)$ & 1.04 & $(0.33,3.26)$ & 0.87 & $(0.25,3.01)$ & 0.87 & $(0.25,3.02)$ & 0.88 & $(0.27,2.86)$ \\
\hline $26-50 \%$ & 0.75 & $(0.26,2.19)$ & 0.79 & $(0.30,2.11)$ & 0.72 & $(0.27,1.97)$ & 0.73 & $(0.28,1.92)$ & 0.77 & $(0.30,1.96)$ \\
\hline $51-75 \%$ & 0.52 & $(0.26,1.02)$ & 0.51 & $(0.27,0.97)$ & 0.56 & $(0.30,1.06)$ & 0.56 & $(0.29,1.08)$ & 0.56 & $(0.28,1.14)$ \\
\hline $76-100 \%$ & REF & REF & REF & REF & REF & REF & REF & REF & REF & REF \\
\hline \multicolumn{11}{|c|}{ Supermarket in neighborhood } \\
\hline Yes & 0.73 & $(0.34,1.44)$ & 0.71 & $(0.39,1.26)$ & 0.68 & $(0.37,1.24)$ & 0.68 & $(0.37,1.25)$ & 0.70 & $(0.36,1.36)$ \\
\hline No & REF & REF & REF & REF & REF & REF & REF & REF & REF & REF \\
\hline \multicolumn{11}{|l|}{ Park in neighborhood } \\
\hline Yes & 0.90 & $(0.44,1.82)$ & 0.89 & $(0.46,1.70)$ & 0.88 & $(0.50,1.56)$ & 0.91 & $(0.51,1.63)$ & 1.03 & $(0.60,1.78)$ \\
\hline No & REF & REF & REF & REF & REF & REF & REF & REF & REF & REF \\
\hline
\end{tabular}

${ }^{a}$ Each SEP measure additionally adjusted for marital status, education, and/or occupation

${ }^{\mathrm{b}}$ Each SEP measure additionally adjusted for public support, house value, and/or family income

${ }^{\mathrm{c}}$ Each SEP measure additionally adjusted for neighborhood supermarket and/or park

${ }^{\mathrm{d}}$ Each SEP measure additionally adjusted for physical activity, smoking, and alcohol intake

continues throughout adulthood [34], is a possible contributing factor in lowering the risk of obesity among low SEP African-American and Caribbean-Black men. This is a hypothesis that should be tested in prospective research. Moreover, the higher obesity rate among high SEP men has been postulated to be due to the positive effect increased body weight has on higher earned income and social prestige among men [35]. Future longitudinal investigations should determine whether perceived and actual positive economic consequences for increasing body size drive the positive relationship between SEP and obesity among men.

For African-American women, the expected inverse relationship between individual-level SEP and obesity was seen for education [15]; however, educational attainment was not associated with obesity among Caribbean-Black women. In line with previous research [36], our study also reveals that higher status occupations, i.e., sales and administrative jobs among African-American women and professional and managerial jobs among Caribbean-Black women, are associated with lower odds for obesity than lesser status service professions. Finally, our results at family-level SEP show that similar to Caribbean-Black men, receipt of public assistance increased odds for obesity among women, with a stronger association for Caribbean-Black women.

Lastly, our results show important ethnic and sex differences in the association between community SEP characteristics and obesity. The lower odds of obesity among Caribbean-Black male and African-American female residents in neighborhoods containing a supermarket is consistent with prior research [23, 37]. In addition, the lower odds of obesity for residence within a neighborhood containing a supermarket found among Caribbean-Black women suggests that this relationship may hold for them as well despite the lack of statistical significance most 
likely due to low statistical power. The absence of any relationship between obesity and residence within a neighborhood containing a supermarket among AfricanAmerican men warrants further investigation.

It is important to highlight the substantial decrease in the odds of obesity exclusively among African-American men who live in neighborhoods containing a park. Research has shown that increased access to parks is associated with increased moderate-to-vigorous physical activity in boys but not among girls aged 8 to 12 years [38]. For many Black men, these childhood patterns of engaging in vigorous competitive team sports (e.g., basketball) in neighborhood parks extend into early adulthood, a pattern not seen historically among Black women [39, 40]. Additional research is needed to determine whether elevated energy expenditure due to increased access to recreational parks across the life course explains the lower prevalence of obesity among AfricanAmerican men living in neighborhoods with community parks. Previous research documenting a lack of association between SEP and exercise frequency in Black women may explain the absence of a relationship between neighborhood parks and obesity among African-American and Caribbean women within our findings [41]. Further theoretical and empirical investigations are needed to uncover additional mechanisms through which measures of SEP at the neighborhood level independently operate to increase obesity within the various ethnic and sex groups.

To our knowledge, this is the first study to document associations between multilevel and multidimensional measures of SEP and obesity in a sample of US self-identified and Caribbean self-identified Black Americans, while using an intersectionality approach centered on both within-race ethnic and sex differentiation. Methodological strengths also include the employment of multiple imputation of missing data. This study has several limitations, however. Multiple imputation was not performed on measures with over $40 \%$ of missing data, such as individual-level income, and so, this variable was not included within the analysis in order to minimize bias $[42,43]$. Furthermore, the lower sample size of CaribbeanBlacks within the NSAL dataset limited statistical power to examine any additional variation in the SEP-obesity associations by nativity status, i.e., foreign-born vs. US-born.

The public-use NSAL questionnaire utilized for analysis included only self-reported measures of neighborhood physical characteristics and did not allow for geocoding addresses to include census tract measures, i.e., percentages of families below the poverty, unemployed and/or low-educated adults, and homeowners within a neighborhood, for multilevel modeling. Although the inability to examine objective assessments of neighborhood SEP within hierarchical models is a disadvantage, perceived measures of neighborhood resources have been found in previous studies to be equally robust correlates of obesity as observed neighborhood indicators [44].
The cross-sectional nature of the NSAL study design does not establish a clear, unbiased temporal relationship between multilevel SEP indicators and adult obesity; therefore, neither causality nor direction of the SEP-obesity associations can be ascertained with certainty. The NSAL, one of the few studies that allow for an examination of the socioeconomic correlates of cardio-metabolic health by ethnicity and sex within Black Americans, was conducted in 2001-2003 and hence is an established dataset. Notwithstanding, the stability of SEP indicators over time for Black Americans, including median income, which was $\$ 40,573$ in 2003 and $\$ 41,361$ in 2018, supports the present-day relevancy of our findings [45].

The use of self-reported weight and height to estimate obesity prevalence is also a potential bias. Studies have found that these self-reported measures are reasonably valid and reliable indicators of actual weight and height, although obese individuals tend to underestimate their weight [46-48]. A dilution rather than an exaggeration of the magnitude of our associations is expected however if a systematic underreporting of weight is similar across SEP categories. Statistical adjustment for additional health behaviors related to obesity such as dietary quality could not be made within the analyses since this information is not assessed in the NSAL. In addition, physical activity, crudely assessed within the NSAL as a continuous measure of reported leisure time activity, is a less reliable measure of activity level than other methods which directly monitor the intensity of physical activity such as the use of double isotopically labeled water $\left(\mathrm{D}_{2} \mathrm{O}^{18}\right)$ methodology and measurement by a pedometer or tachometer [49]. The extent to which the inclusion of dietary intake and a more reliable indicator of the intensity of physical activity would explain the associations between multilevel indicators of SEP and obesity cannot be determined.

Our study findings highlight that adulthood SEP at the individual, family, and neighborhood levels plays an important role in obesity in the US Black population, and this varies by ethnicity and sex. The opposite SEP-obesity relationships between Black men and women suggest the need for further empirical investigation into the sex-specific mechanisms of obesity, particularly among low SEP families where the disparity between young adult Black women and men is the largest [50]. Moreover, the lower odds of obesity associated with residence in neighborhoods with a supermarket for African-American women and Caribbean-Black men, and with residence in neighborhoods containing a park for African-American men, suggests that continued public policy attention to increasing access to food and recreational facilities within Black American communities, as was undertaken in the national "Let us Move!" comprehensive initiative for preventing childhood obesity [51], is likely to be an important endeavor. Evidence from this study also suggests that the development of targeted, multilevel, cultural, and sex-specific societal interventions could be vital to 
not only curtailing the US obesity epidemic but to reducing racial inequities in survival from emerging infectious diseases such as COVID-19, for which obesity has arisen as a strong risk and prognostic factor in its severity [52-54].

Funding Information This study was funded by the Robert Wood Johnson Health and Society Scholar's Program and by the National Institute on Minority Health and Health Disparities (grant number K22MD006133).

\section{Compliance with Ethical Standards}

Conflict of Interest The authors declare that they have no conflicts of interest.

Ethical Approval All procedures performed in studies involving human participants were in accordance with the ethical standards of the institutional research committee and with the 1964 Helsinki Declaration and its later amendments or comparable ethical standards.

Informed Consent Informed consent was obtained from all individual participants included in the study.

\section{References}

1. Villarroel MA, Blackwell DL, Jen A. Tables of Summary Health Statistics for U.S. Adults: 2018 National Health Interview Survey, in National Center for Health Statistics 2019.

2. Sobal J, Stunkard AJ. Socioeconomic status and obesity: a review of the literature. Psychol Bull. 1989;105(2):260-75.

3. Stunkard AJ. Socioeconomic status and obesity. Ciba Found Symp. 1996;201:174-82 discussion 182-7, 188-93.

4. McLaren L. Socioeconomic status and obesity. Epidemiol Rev. 2007;29:29-48.

5. Wang Y, Beydoun MA. The obesity epidemic in the United Statesgender, age, socioeconomic, racial/ethnic, and geographic characteristics: a systematic review and meta-regression analysis. Epidemiol Rev. 2007;29:6-28.

6. Lynch JW, Kaplan GA. In: Berkman LF, Kawachi I, editors. Socioeconomic position in Social Epidemiology. New York: Oxford University Press; 2000. p. 13-35.

7. Krieger N, Williams DR, Moss NE. Measuring social class in US public health research: concepts, methodologies, and guidelines. Annu Rev Public Health. 1997;18:341-78.

8. Williams DR, Collins C. US socioeconomic and racial-differences in health - patterns and explanations. Annu Rev Sociol. 1995;21: 349-86.

9. Lovasi GS, Hutson MA, Guerra M, Neckerman KM. Built environments and obesity in disadvantaged populations. Epidemiol Rev. 2009;31(1):7-20.

10. Rundle A, Quinn J, Lovasi G, Bader MDM, Yousefzadeh P, Weiss $\mathrm{C}$, et al. Associations between body mass index and park proximity, size, cleanliness, and recreational facilities. Am J Health Promot. 2013;27(4):262-9.

11. National Center for Education Statistics. Improving the Measurement of Socioeconomic Status for the National Assessment of Educational Progress: A Theoretical Foundation. 2012.

12. Zhang Q, Wang Y. Trends in the association between obesity and socioeconomic status in U.S. adults: 1971 to 2000. Obes Res. 2004;12(10):1622-32.
13. Flegal KM, Harlan WR, Landis JR. Secular trends in body mass index and skinfold thickness with socioeconomic factors in young adult women. Am J Clin Nutr. 1988;48(3):535-43.

14. Zhang Q, Wang Y. Socioeconomic inequality of obesity in the United States: do gender, age, and ethnicity matter? Soc Sci Med. 2004;58(6):1171-80.

15. Ogden CL, et al. Obesity and socioeconomic status in adults: United States, 2005-2008. NCHS Data Brief. 2010(50):1-8.

16. Mujahid MS, Diez Roux AV, Borrell LN, Nieto FJ. Cross-sectional and longitudinal associations of BMI with socioeconomic characteristics. Obes Res. 2005;13(8):1412-21.

17. Assari $\mathrm{S}$, et al. Race by gender group differences in the protective effects of socioeconomic factors against sustained health problems across five domains. J Racial Ethn Health Disparities. 2016.

18. Flegal KM, Harlan WR, Landis JR. Secular trends in body mass index and skinfold thickness with socioeconomic factors in young adult men. Am J Clin Nutr. 1988;48(3):544-51.

19. Barrington DS, Baquero MC, Borrell LN, Crawford ND. Racial/ ethnic disparities in obesity among US-born and foreign-born adults by sex and education. Obesity (Silver Spring). 2010;18(2):422-4.

20. Heeringa SG, Wagner J, Torres M, Duan N, Adams T, Berglund P. Sample designs and sampling methods for the collaborative psychiatric epidemiology studies (CPES). Int J Methods Psychiatr Res. 2004;13(4):221-40.

21. Pennell B-E, Bowers A, Carr D, Chardoul S, Cheung GQ, Dinkelmann K, et al. The development and implementation of the National Comorbidity Survey Replication, the National Survey of American Life, and the National Latino and Asian American Survey. Int J Methods Psychiatr Res. 2004;13(4):241-69.

22. National Heart, Lung and Blood Institute. Clincal Guidelines on the Identification, Evaluation, and Treatment of Overweight and Obesity in Adults. 1998: Bethesda.

23. Sullivan SM, Brashear MM, Broyles ST, Rung AL. Neighborhood environments and obesity among Afro-Caribbean, African American, and non-Hispanic white adults in the United States: results from the National Survey of American Life. Prev Med. 2014;61:1-5.

24. Kahn HS, Williamson DF. Is race associated with weight change in US adults after adjustment for income, education, and marital factors? Am J Clin Nutr. 1991;53(6 Suppl):1566S-70S.

25. Klesges RC, Meyers AW, Klesges LM, LaVasque ME. Smoking, body weight, and their effects on smoking behavior: a comprehensive review of the literature. Psychol Bull. 1989;106(2):204-30.

26. Raghunathan TE, Solenberger PW, Van Hoewyk JV. IVEware: Imputation and variation estimation software, Survey Research Center, Institute for Social Research, University of Michigan. 2002.

27. Raghunathan $\mathrm{T}$, et al. A multivariate technique for multiply imputing missing values using a sequence of regression models. Surv Methodol. 2001;27(1):85-95.

28. SAS Institute Inc. SAS/STAT 9.1 User's Guide. 2004: Cary.

29. Research Triangle Institute. SUDAAN Language Manual, Release 9.0. 2004: Research Triangle Park.

30. Leung CW, Villamor E. Is participation in food and income assistance programmes associated with obesity in California adults? Results from a state-wide survey. Public Health Nutr. 2011;14(4): $645-52$.

31. Bennett GG, Wolin KY, James SA. Lifecourse socioeconomic position and weight change among blacks: the Pitt County study. Obesity (Silver Spring). 2007;15(1):172-81.

32. Croft JB, Strogatz DS, James SA, Keenan NL, Ammerman AS, Malarcher AM, et al. Socioeconomic and behavioral correlates of body mass index in black adults: the Pitt County study. Am J Public Health. 1992;82(6):821-6.

33. Harrell JS, et al. Leisure time activities of elementary school children. Nurs Res. 1997;46(5):246-53. 
34. Stamps SM, Stamps MB. Race, class and leisure activities of urban residents. J Leis Res. 1985;17(1):40-56.

35. McLean RA, Moon M. Health, obesity, and earnings. Am J Public Health. 1980;70(9):1006-9.

36. Averett S, Korenman S. Black-white differences in social and economic consequences of obesity. Int J Obes Relat Metab Disord. 1999;23(2):166-73.

37. Lopez RP. Neighborhood risk factors for obesity. Obesity (Silver Spring). 2007;15(8):2111-9.

38. Roemmich JN, Epstein LH, Raja S, Y in L. The neighborhood and home environments: disparate relationships with physical activity and sedentary behaviors in youth. Ann Behav Med. 2007;33(1):2938 .

39. Floyd MF, Spengler JO, Maddock JE, Gobster PH, Suau LJ. Parkbased physical activity in diverse communities of two U.S. cities. An observational study. Am J Prev Med. 2008;34(4):299-305.

40. Cohen DA, McKenzie TL, Sehgal A, Williamson S, Golinelli D, Lurie N. Contribution of public parks to physical activity. Am J Public Health. 2007;97(3):509-14.

41. Assari S. Educational attainment and exercise frequency in American women; Blacks' diminished returns. Womens Health Bull. 2019;6(3).

42. Dong YR, Peng CYJ. Principled missing data methods for researchers. Springerplus. 2013;2:17.

43. Jakobsen JC, et al. When and how should multiple imputation be used for handling missing data in randomised clinical trials - a practical guide with flowcharts. BMC Med Res Methodol. 2017;17:10.

44. Boehmer TK, Hoehner CM, Deshpande AD, Brennan Ramirez LK, Brownson RC. Perceived and observed neighborhood indicators of obesity among urban adults. Int J Obes. 2007;31(6):968-77.

45. Households by Total Money Income, Race, and Hispanic Origin of Householder: 1967 to 2018. Available at census.gov (Census Bureau).
46. Stewart AL. The reliability and validity of self-reported weight and height. J Chronic Dis. 1982;35(4):295-309.

47. Rowland ML. Self-reported weight and height. Am J Clin Nutr. 1990;52(6):1125-33.

48. Gillum RF, Sempos CT. Ethnic variation in validity of classification of overweight and obesity using self-reported weight and height in American women and men: the Third National Health and Nutrition Examination Survey. Nutr J. 2005;4:27.

49. Erlichman J, Kerbey AL, James WP. Physical activity and its impact on health outcomes. Paper 2: prevention of unhealthy weight gain and obesity by physical activity: an analysis of the evidence. Obes Rev. 2002;3(4):273-87.

50. Robinson WR, Gordon-Larsen P, Kaufman JS, Suchindran CM, Stevens J. The female-male disparity in obesity prevalence among black American young adults: contributions of sociodemographic characteristics of the childhood family. Am J Clin Nutr. 2009;89(4): 1204-12.

51. White House Task Force on Childhood Obesity Report to the President. Solving the Problem of Childhood Obesity Within a Generation. 2010: Washington.

52. Azar KMJ, et al. Disparities In Outcomes Among COVID-19 Patients In A Large Health Care System In California. Health Aff (Millwood). 2020:101377hlthaff202000598.

53. Fouad MN, Ruffin J, Vickers SM. COVID-19 is Out of Proportion in African Americans. This Will Come as No Surprise.... Am J Med. 2020.

54. Tamara A, Tahapary DL. Obesity as a predictor for a poor prognosis of COVID-19: a systematic review. Diabetes Metab Syndr. 2020;14(4):655-9.

Publisher's Note Springer Nature remains neutral with regard to jurisdictional claims in published maps and institutional affiliations. 